\title{
UNA ESPECIE NUEVA DE BURSERA (BURSERACEAE) DEL ESTADO DE OAXACA (MÉXICO)*
}

JERZY RZEDOWSKI

Y

\author{
Graciela Calderón de Rzedowski \\ Instituto de Ecología, A.C. \\ Centro Regional del Bajío \\ Apartado postal 386 \\ 61600 Pátzcuaro, Michoacán
}

\begin{abstract}
RESUMEN
Se describe como nueva y se ilustra a Bursera laurihuertae, árbol que es un componente importante del bosque tropical caducifolio del sector SE del estado de Oaxaca. La especie forma parte de la sección Bursera, pero se destaca por sus hojas predominantemente simples. Se le compara con B. schlechtendalii Engl., con B. instabilis McVaugh \& Rzed. y con B. lunanii (Spreng.) Adams \& Dandy, pero sin poder concluir acerca de su más cercano parentesco.
\end{abstract}

\section{ABSTRACT}

Bursera laurihuertae, an important tree element of the tropical deciduous forests of the southeastern part of the Mexican state of Oaxaca, is described and illustrated. The species is a member of section Bursera, but stands out because of its predominantly simple leaves. It is compared with B. schlechtendalii Engl., with B. instabilis McVaugh \& Rzed., as well as with the Jamaican B. Iunanii (Spreng.) Adams \& Dandy, but no firm conclusion can be drawn concerning its close phylogenetic relationship.

Exploraciones botánicas realizadas en el estado de Oaxaca revelan la existencia de varios componentes del género Bursera que aún no han sido dados a conocer. Uno de ellos se describe a continuación.

Bursera laurihuertae Rzedowski \& Calderón sp. n. Fig. 1.

Arbor usque 8(15) $\mathrm{m}$ alta ut videtur dioecia glabra; truncus usque $30 \mathrm{~cm}$ diametro cortice exteriore papyraceo rubello exfolianti; folia maximam partem simplicia (unifoliolata)

* Trabajo realizado con apoyo económico del Instituto de Ecología, A.C. (cuenta 902-03), del Consejo Nacional de Ciencia y Tecnología, así como de la Comisión Nacional para el Conocimiento y Uso de la Biodiversidad. 
plerumque obovata usque $6(9) \mathrm{cm}$ longa et $4(4.5) \mathrm{cm}$ lata apice truncata vel rotundata margine integra basi cuneata discolora chartacea vel coriacea, aliqua trifoliolata, rarissime 5-foliolata; inflorescentiae masculae racemiformes vel paniculatae, flores masculi pentameri, calycis lobi late triangulares $0.2-0.3 \mathrm{~mm}$ longi, petala oblongo-elliptica $2-3 \mathrm{~mm}$ longa, stamina 10; inflorescentiae femineae racemiformes vel interdum uniflorae, flores feminei tetrameri masculinorum similes sed petalis latioribus, staminodia 8, ovarium triloculare, stylus brevissimus; drupae trivalvatae oblique ovoideae 9-11 $\mathrm{mm}$ longae apice acutae, pyrenae oblique ovoideae pseudoarillo roseo omnino indutae.

Árbol caducifolio, aparentemente dioico, resinoso y aromático al estrujarse, hasta de 8(15) $\mathrm{m}$ de alto, glabro, de copa amplia; tronco hasta de $30 \mathrm{~cm}$ de diámetro, su corteza externa rojiza, exfoliante en tiras grandes y delgadas; hojas concentradas en los ápices de ramillas cortas, o bien, alternas y espaciadas en ramillas largas de crecimiento nuevo, peciolos hasta de $1.5(3.5) \mathrm{cm}$ de largo, con frecuencia todas simples (unifolioladas), con lámina angosta a ampliamente obovada, rara vez oblonga, hasta de 6(9) cm de largo y 4(4.5) $\mathrm{cm}$ de ancho, redondeada o más frecuentemente truncada, a menudo apiculada o cuspidada, pero no pocas veces retusa en el ápice, margen entero, base por lo general cuneada, pero llegando a ser redondeada a truncada, de consistencia cartácea a coriácea, verde oscura en el haz, mucho más clara en el envés, en ocasiones algunas ramillas llevan también hojas trifolioladas y excepcionalmente 5-folioladas, en las cuales el foliolo terminal es similar en forma a la hoja unifoliolada, mientras que los laterales tienden a ser elípticos y con frecuencia son de base redondeada y más o menos oblicua, los peciólulos miden 1 a $3 \mathrm{~mm}$ de largo y el raquis carece de alas; inflorescencias masculinas racemiformes o paniculadas, hasta de $6 \mathrm{~cm}$ de largo, flores masculinas pentámeras, lóbulos del cáliz anchamente triangulares, de 0.2 a $0.3 \mathrm{~mm}$ de largo, pétalos oblongo-elípticos, de 2 a $3 \mathrm{~mm}$ de largo, agudos en el ápice, estambres 10, todos semejantes, anteras oblongas, de ca. $0.7 \mathrm{~mm}$ de largo, gineceo rudimentario o ausente; inflorescencias femeninas racemiformes, hasta de $4 \mathrm{~cm}$ de largo, con cierta frecuencia reducidas a una sola flor, ésta tetrámera, similar a la masculina, pero con los pétalos un poco más anchos, tendiendo a ovados, estaminodios 8, sus anteras de ca. $0.5 \mathrm{~mm}$ de largo, ovario trilocular, estilo muy corto; drupas trivalvadas, oblicuamente ovoides, de 9 a $11 \mathrm{~mm}$ de largo y 5 a $6 \mathrm{~mm}$ de diámetro, agudas en el ápice, hueso oblicuamente ovoide, de 7 a $9 \mathrm{~mm}$ de largo, totalmente cubierto por el pseudoarilo de color rosado.

TIPO: México, Oaxaca: $3 \mathrm{~km}$ al NE de San Juan Guegoyache, municipio de Totolapan, alt. $950 \mathrm{~m}$, ladera de roca ígnea con vegetación de bosque tropical caducifolio, 9.VIII.1976, J. Rzedowski 34383 (holotipo IEB, isotipos por distribuirse a ANSM, CAS, CHAPA, CIIDIR, ENCB, IBUG, MEXU, MICH, NY, OAX, TEX, XAL).

Material adicional examinado (la información correspondiente a los ejemplares depositados en NY fue amablemente proporcionada por el Dr. D. Daly): cerca de Las Fuentes, $8 \mathrm{~km}$ al W de Marilú, municipio de Magdalena Tequisistlán, S. Acosta C. 1360 (IEB, MEXU); $7 \mathrm{~km}$ del poblado La Chiviza al rancho Nhizavigena, carretera a Santiago Loallaga, municipio de Santa María Guienagati, J. I. Calzada 19298 (MEXU, NY); Barra de la Cruz, 1.5 km SE, laguna del cerrito, 1549'59' N, 9557’25' W, municipio de Santiago Astata, M. Elorsa 2134 (IEB); camino Pino Suárez - Duraznillo, municipio de Totolapan, A. 


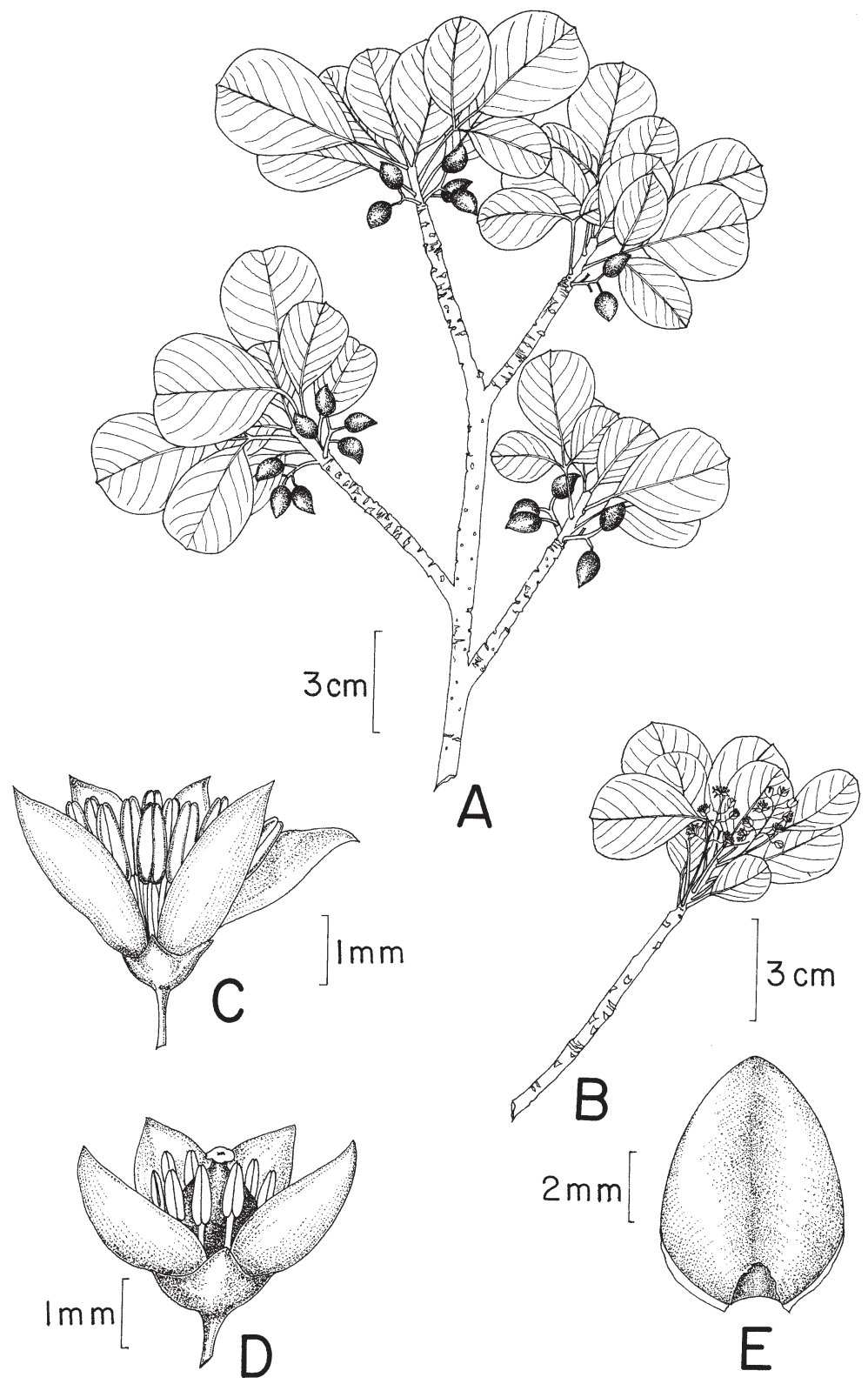

Bursera laurihuertae Rzed. \& Calderón. A. rama con frutos; B. rama con flores; C. flor masculina; D. flor femenina; E. hueso. Ilustrado por Alfonso Barbosa. 
Flores M. 1335 (ENCB, IEB); Guiengola, municipio de Tehuantepec, T. MacDougall 5 (MEXU); Llano Copal, Tequisistlán, municipio de Magdalena Tequisistlán, T. MacDougall 16 (MEXU); El Cerro Arenal, al N de Buenos Aires, municipio de Buenos Aires, C. Martínez R. 13 (IEB); El Cerro Cuachi, al O de Salina Cruz, municipio de Salina Cruz, C. Martínez R. 168 (IEB); San Vicente Mazatán, 20 km al N del Morro, entrando por el crucero Santa Clara, 160.' N, 95²5' W, municipio de Salina Cruz, C. Martínez 1561 (IEB); El Camarón, ca. 35 miles west of Tehuantepec, along the Panamerican highway between Oaxaca and Tuxtla Gutierrez, municipio de Nexapa, R. McVaugh 21859 (ENCB); Tehuantepec - Juchitán, F. Miranda 8476 (MEXU); cerca de Juchitán, municipio de Juchitán, F. Miranda 8547 (MEXU); $2 \mathrm{~km}$ al N de La Ceiba, municipio de Totolapan, J. Rzedowski 34391 (IEB); 3 km al NW de El Camarón, sobre la carretera a Oaxaca, municipio de Nexapa, J. Rzedowski 35428 (IEB); 3 km al NE de San Juan Guegoyache, municipio de Totolapan, J. Rzedowski 35649 (IEB); cerca de La Chivera, 8 km al SE de Santa María Guienagati, sobre el camino a Ixtepec, municipio de Santa María Guienagati, J. Rzedowski 38137 (IEB); 9 km al SE de Totolapan, rumbo a Zoquitlán, municipio de Totolapan, A. Saynes 1197 (IEB, MEXU); a $10 \mathrm{~km}$ de Juchitán, rumbo a Tehuantepec, municipio de Juchitán, A. Saynes V. 1551 (ENCB, IEB, MEXU, NY); río Cotita, al NO de Tehuantepec, municipio de Tehuantepec, $R$. Torres 8371 (IEB, MEXU); rancho Ricardo, al N de Buenos Aires, entrando por Hierba Santa, municipio de Buenos Aires, R. Torres y C. Martínez 7308 (IEB); Cerro de la Cueva de Lleza, municipio de Tehuantepec, F. Ventura 16446 (IEB); cerro Guiengola, municipio de Tehuantepec, F. Ventura 16450 (IEB); El Camarón, municipio de Nexapa, F. Ventura 16518 (IEB); San José de Gracia, municipio de Totolapan, F. Ventura 16525 (IEB); Torre de microondas, municipio de Jalapa de Marqués, F. Ventura 16538 (IEB); cerca de la presa, municipio de Jalapa de Marqués, F. Ventura 16541 (IEB); El Salado, municipio de Magdalena Tequisistlán, F. Ventura 16550 (IEB); Marilú, municipio de Magdalena Tequisistlán, F. Ventura 16554 (IEB); cerca de la Esc. Técnica Agropecuaria, municipio de Totolapan, F. Ventura 16575 (IEB); Mano del León, municipio de Santa María Zoquitlán, F. Ventura 16579 (IEB); 35 mi. SE of Jct. of road to Mitla and Hwy. 190, municipio de Totolapan, Wallace et al. 459 (MEXU, NY); Huazantlán del Río, Cerro de Huazantlán, ladera sur, municipio de San Mateo del Mar, D. Zizumbo y P. Colunga 306 (MEXU).

La especie parece estar restringida en su distribución al sector SE del estado de Oaxaca, donde a menudo forma parte importante del bosque tropical caducifolio en altitudes entre 0 y $1400 \mathrm{~m}$. Se le ha registrado con particular abundancia en la cuenca del río Tehuantepec y también es frecuente en las porciones bajas de la franja meridional del Istmo, donde comparte con B. simaruba (L.) Sarg. el nombre común de "mulato". En la región indígena huave se le denomina "jit kiek" (comida de pájaro), se le emplea en cercas vivas y tiene usos medicinales.

Su época de floración corresponde a los meses de marzo y abril, cuando la mayor parte de las veces los árboles se encuentran sin hojas.

En el cuadro 1 se comparan algunas características de aparente mayor significación entre $B$. laurihuertae y las restantes especies conocidas de México, con corteza exfoliante roja o rojiza (pertenecientes a la sección Bursera), que presentan al menos algunas hojas unifolioladas.

Como lo enfatizaron Rzedowski y Kruse (1979), la tendencia a la reducción del número de foliolos es un carácter que se ha manifestado independientemente en diversos linajes dentro del género. Así, B. crenata P. G. Wilson y B. trimera Bullock (al igual que 
probablemente también B. rzedowskii Toledo), que tienen las hojas finamente crenadoserradas, definen una línea separada que, de acuerdo con el nuevo esquema filogenético propuesto por Becerra y Venable (1999), forman parte del conjunto llamado "fragilis", que incluye entre otras especies a B. kerberi Engl., B. multijuga Engl. y B. lancifolia (Schlecht.) Engl.

A su vez, aunque con ciertas reservas, estos últimos autores ubican a $B$. schlechtendalii Engl. y a $B$. chemapodicta Rzed. \& Ortiz en el grupo que denominan "fagaroides", a un lado de B. fagaroides (H.B.K.) Engl., B. ariensis (H.B.K.) McVaugh \& Rzed. y de $B$. aptera Ramírez.

Cuadro 1. Comparación de algunos caracteres de Bursera laurihuertae con los de otras especies mexicanas de Bursera de corteza exfoliante roja o rojiza y hojas, al menos ocasionalmente unifolioladas.

\begin{tabular}{|c|c|c|c|c|c|c|}
\hline & $\begin{array}{l}\text { Número de } \\
\text { foliolos }\end{array}$ & $\begin{array}{c}\text { Forma de la } \\
\text { hoja } \\
\text { unifoliolada }\end{array}$ & $\begin{array}{l}\text { Margen de } \\
\text { la lámina } \\
\text { foliar }\end{array}$ & $\begin{array}{c}\text { Pubescencia } \\
\text { de la hoja }\end{array}$ & Infrutescencia & $\begin{array}{l}\text { Forma y } \\
\text { largo del } \\
\text { fruto }\end{array}$ \\
\hline B. chemapodicta & 1 & $\begin{array}{l}\text { ovada a } \\
\text { obovada }\end{array}$ & entero & presente & $\begin{array}{c}\text { frutos } \\
\text { solitarios a } \\
\text { fasciculados }\end{array}$ & $\begin{array}{l}\text { subglobosa } \\
\text { a obovoide } \\
5 \text { a } 7 \mathrm{~mm}\end{array}$ \\
\hline B. crenata & 1 & $\begin{array}{c}\text { oblanceola- } \\
\text { da a elíptica } \\
\text { u oblonga }\end{array}$ & $\begin{array}{c}\text { finamente } \\
\text { crenado- } \\
\text { serrado }\end{array}$ & ausente & $\begin{array}{c}\text { frutos } \\
\text { solitarios o } \\
\text { en cimas }\end{array}$ & $\begin{array}{l}\text { oblicuamen- } \\
\text { te obovoide } \\
6 \text { a } 7 \mathrm{~mm}\end{array}$ \\
\hline B. instabilis & $1,3(5)$ & $\begin{array}{c}\text { ovada a } \\
\text { lanceolada } \\
\text { o elíptica }\end{array}$ & entero & $\begin{array}{l}\text { presente } \\
\text { en la base } \\
\text { del envés }\end{array}$ & $\begin{array}{l}\text { frutos en } \\
\text { racimos o } \\
\text { solitarios }\end{array}$ & $\begin{array}{l}\text { oblicuamen- } \\
\text { te obovoide } \\
6 \text { a } 8 \mathrm{~mm}\end{array}$ \\
\hline B. krusei & $(1,2) 3$ & $\begin{array}{c}\text { lanceolada } \\
\text { a ovada }\end{array}$ & entero & presente & $\begin{array}{l}\text { frutos en } \\
\text { racimos o } \\
\text { panículas }\end{array}$ & $\begin{array}{c}\text { ovoide a } \\
\text { subglobosa } \\
6 \text { a } 8 \mathrm{~mm}\end{array}$ \\
\hline B. laurihuertae & $1,3(5)$ & $\begin{array}{l}\text { obovada a } \\
\text { elíptica }\end{array}$ & entero & ausente & $\begin{array}{l}\text { frutos en } \\
\text { racimos o } \\
\text { solitarios }\end{array}$ & $\begin{array}{l}\text { oblicuamen- } \\
\text { te ovoide } \\
9 \text { a } 11 \mathrm{~mm}\end{array}$ \\
\hline B. rzedowskii & 1 & $\begin{array}{c}\text { elíptica a } \\
\text { oblonga u } \\
\text { oblanceola- } \\
\text { da }\end{array}$ & $\begin{array}{l}\text { subentero a } \\
\text { inconspicua } \\
\text { mente serru } \\
\text { lado }\end{array}$ & ausente & $\begin{array}{c}\text { frutos } \\
\text { solitarios o } \\
\text { fasciculados }\end{array}$ & $\begin{array}{c}\text { subglobosa } \\
\text { a ovoide } \\
5 \text { a } 8 \mathrm{~mm}\end{array}$ \\
\hline B. schlechtendalii & 1 & $\begin{array}{l}\text { elíptica a } \\
\text { obovada }\end{array}$ & entero & ausente & $\begin{array}{c}\text { frutos } \\
\text { solitarios o } \\
\text { fasciculados }\end{array}$ & $\begin{array}{l}\text { oblicuamen- } \\
\text { te ovoide } \\
4 \text { a } 8 \mathrm{~mm}\end{array}$ \\
\hline B. trimera & (1) 3 & $\begin{array}{c}\text { elíptica a } \\
\text { oblonga u } \\
\text { oblanceola- } \\
\text { da }\end{array}$ & $\begin{array}{l}\text { finamente } \\
\text { serrado }\end{array}$ & ausente & $\begin{array}{c}\text { frutos } \\
\text { solitarios o } \\
\text { fasciculados }\end{array}$ & $\begin{array}{l}\text { subglobosa } \\
\text { a oblicua- } \\
\text { mente obo- } \\
\text { voide } \\
5 \text { a } 7 \mathrm{~mm}\end{array}$ \\
\hline
\end{tabular}


La afinidad de $B$. instabilis McVaugh \& Rzed. con el conjunto llamado "simaruba" queda confirmada también con el análisis de ADN realizado por Becerra y Venable (op. cit.) y en función de sus rasgos morfológicos es probable que $B$. krusei Rzed. (que no fue muestreada para el mencionado estudio) igualmente pertenezca aquí.

Las características de la hoja de $B$. laurihuertae $(\mathrm{BI})$ son muy semejantes a los de $B$. schlechtendalii (Bs) y muchos ejemplares que se mencionan en la lista que antecede habían sido identificados y distribuidos con este último nombre. La similitud llega a ser tan grande, que a veces resulta difícil la identificación de ejemplares totalmente unifoliolados y estériles, procedentes del área en que ambas especies conviven.

Sin embargo, los dos taxa discrepan formalmente en los siguientes caracteres:

1. BI desarrolla con mucha frecuencia hojas trifolioladas, particularidad que no se ha observado en Bs en ninguna de las poblaciones de su amplia área de distribución, que se extiende desde el norte de México hasta la Península de Yucatán y Guatemala.

2. Las flores masculinas y frecuentemente las femeninas de $\mathrm{BI}$ se agrupan en inflorescencias ramificadas, mientras que las de Bs nacen solitarias, fasciculadas o subfasciculadas.

3. Los frutos maduros y frescos de $\mathrm{BI}$ miden 9 a $11 \mathrm{~mm}$ de largo y se insertan sobre pedicelos y pedúnculos que por lo general son rectos y en total por lo común miden $6 \mathrm{~mm}$ o más de longitud; en cambio los frutos de Bs alcanzan sólo 4 a $8 \mathrm{~mm}$ de largo y sus pedúnculos, casi siempre notablemente encorvados, son de menos de $5 \mathrm{~mm}$ de longitud.

4. Otros rasgos de más difícil descripción y cuantificación son:

a) la corteza externa exfoliante en $\mathrm{Bl}$ es de color más claro que en Bs;

b) la base del fruto en BI es truncada, a diferencia de Bs, donde suele ser redondeada u obtusa.

Con el conjunto de las mencionadas diferencias no es seguro de que exista un vínculo filogenético cercano entre las dos especies y se plantea la posibilidad de que la similitud en la morfología foliar puede obedecer a un proceso evolutivo de convergencia.

Otra especie con la que cabe hacer comparación es $B$. instabilis que extiende su área de distribución a lo largo del litoral pacífico de Jalisco a Oaxaca. Esta última suele diferir en forma notable de $B$. laurihuertae en sus hojas (o foliolos) ovadas a lanceoladas o elípticas, agudas a acuminadas en el ápice, de textura más delgada y pubescentes en la parte basal del envés, así como en las inflorescencias más largas y ramificadas y en los frutos más encorvados.

Sin embargo, al revisar una extensa serie de ejemplares de herbario de $B$. instabilis, los que escriben han podido observar una extraordinaria variabilidad que atañe tanto a la forma de las hojas, como de inflorescencias y de frutos, de suerte que, salvo el carácter de la pubescencia, ninguno de los mencionados resulta plenamente contrastante.

En consecuencia, tampoco procedería excluir en forma definitiva la posibilidad de una relación de parentesco relativamente próximo.

Por otro lado, en las islas del archipiélago antillano (así como en Venezuela) crecen también especies pertenecientes a la sección Bursera con corteza rojiza exfoliante y varias se han descrito con hojas uni o uni a trifolioladas de margen entero. De este grupo (que no ha sido estudiado por Becerra y Venable (op. cit.)) y en virtud de sus hojas elípticas 
a obovadas, la planta jamaiquina B. lunanii (Spreng.) Adams \& Dandy (antes conocida como B. simplicifolia DC.) tiene bastante parecido con B. laurihuertae. No obstante, la primera se caracteriza por sus hojas escasamente discoloras y de peciolo más grueso, sus flores (al menos las masculinas) se agrupan en panículas racemiformes relativamente largas, son muy pequeñas (de escasos $2 \mathrm{~mm}$ de longitud) y tienen los lóbulos del cáliz de aproximadamente la mitad del largo de los pétalos, mientras que los frutos (de acuerdo con la información amablemente proporcionada por el Dr. D. Daly) son aún más grandes que los de $B$. laurihuertae. De lo anterior cabe deducir que posiblemente esta similitud es también de tipo convergente y no refleja clara relación de origen común cercano.

El nombre de este taxon se dedica para honrar la memoria de la recientemente desaparecida botánica mexicana Laura Huerta Múzquiz (1913-2000), destacada estudiosa de la ficoflora de los litorales de nuestro país.

\section{LITERATURA CITADA}

Becerra, J. y D. L. Venable. 1999. Nuclear ribosomal DNA phylogeny and its implications for evolutionary trends in Mexican Bursera (Burseraceae). Amer. Journ. Bot. 86: 1047-1057.

Rzedowski, J. y H. Kruse. 1979. Algunas tendencias evolutivas en Bursera (Burseraceae). Taxon 28: 103-116. 